\title{
Distinct Serum and Vitreous Inflammation-Related Factor Profiles in Patients with Proliferative Vitreoretinopathy
}

Yao Ni $\cdot$ Yingyan Qin · Zijing Huang · Fangyuan Liu • Shaochong Zhang $\cdot$ Zhaotian Zhang (D)

Received: March 6, 2020 / Published online: April 9, 2020 (C) The Author(s) 2020 or epiretinal membrane (ERM) patients without any other ocular or systemic disease.

Results: Thirty-six serum samples and 34 vitreous samples were obtained. Thirty-one different growth factors and cytokines were detected in serum samples. However, none of the circulating growth factors and cytokines were found to be different from the controls. Ten different growth factors and cytokines were measured in the vitreous samples. The concentration levels of PDGF-AA, TGF- $\alpha$, VEGF, IL-6, IL-8, and TNF $\beta$ were found to have significantly increased in the vitreous of PVR patients.

Conclusion: Our study found that none of the circulating inflammation-related factors were changed in PVR or RRD patients, indicating the absence of a system inflammatory biomarkers to predict the development of proliferative vitreoretinopathy. As a supplement to previous research, the concentrations of PDGF-AA, TGF$\alpha$, VEGF, IL-6, IL-8, and TNF $\beta$ were significantly upregulated in the vitreous of PVR patients. These factors should be considered for preventing PVR.

Keywords: Cytokine; Inflammation; Ophthalmology; Proliferative vitreoretinopathy; Rhegmatogenous retinal detachment; Serum; Vitreous
Y. Ni · Y. Qin · F. Liu · S. Zhang $(\bowtie) \cdot$ Z. Zhang $(\bowtie)$ State Key Laboratory of Ophthalmology, Zhongshan Ophthalmic Center, Sun Yat-Sen University, Guangzhou, China

e-mail: zhshaochong@outlook.comZ. Zhang

e-mail: zhangzht9@mail.sysu.edu.cn

\section{Z. Huang}

Joint Shantou International Eye Center of Shantou University and The Chinese University of Hong

Kong, Shantou, China 


\section{Key Summary Points}

Proliferative vitreoretinopathy (PVR), which is regulated by growth factors and cytokines, is the leading cause of failure in vitreoretinal surgery. Therefore, new therapeutic targets or early detection and monitoring of biomarkers are needed to prevent PVR.

While the role of local retinal inflammation in the development of PVR is documented, we aimed to assess not only the cytokine profile in the vitreous but also the growth factors and cytokine profile in serum to discover new diagnostic markers for evaluating treatment response in PVR patients.

Our study for the first time reported the levels of circulating inflammation-related factors in PVR or RRD patients.

Our findings show that none of the inflammation-related factors were significantly different in serum, indicating that PVR was only a local retinal inflammation.

As a supplement to previous research, the concentrations of PDGF-AA, TGF- $\alpha$, VEGF, IL-6, IL-8, and TNF $\beta$ significantly increased in the vitreous fluid of PVR patients.

\section{INTRODUCTION}

Rhegmatogenous retinal detachment (RRD), which is caused by the detachment of the photoreceptor layer from the retinal pigment epithelium, occurs in about 1 in 20,000 people per year [1]. With the improvement of instrumentation and surgical techniques, the cure rate for RRD has improved. However, 5-10\% of patients with RD may develop proliferative vitreoretinopathy (PVR), which is the leading cause of vitreoretinal surgery failure and severely impairs visual outcome [2].

Disruption of several inflammation-related factors in the vitreous has been found to be associated with the development and progression of PVR [3]. Inflammatory factors could mediate a wound-healing response involving cell migration, proliferation, epithelial-mesenchymal transformation (EMT), and matrix synthesis, which could result in PVR. Growth factors, such as transforming growth factor $\beta-1$ (TGF $\beta-1),-2$, -3, vascular endothelial growth factor (VEGF), and platelet-derived growth factor (PDGF), were found to be significantly upregulated in RRD and PVR patients and proved to be involved in the formation of extracellular matrix (ECM) [4-6]. Other inflammatory cytokines, such as interleukin-6 (IL-6), IL-8, tumor necrosis factor $\alpha$ (TNF $\alpha$ ), monocyte chemotactic protein-1 (MCP-1), macrophage inflammatory protein-1 (MIP-1), and interferoninducible $10-\mathrm{kDa}$ protein (IP-10), were also upregulated in the vitreous of RRD patients [7-9]. The levels of IL- 8 and TGF $\beta-3$ were related to the extent of retinal detachment [5]. The upregulation of growth factors and cytokines, such as TGF $\beta$, VEGF, PDGF, IL-6, IL-8, and $\mathrm{TNF} \alpha$, was proposed as a marker for the formation of PVR [2]. However, no specific factors were found for PVR [10].

Therefore, new therapeutic targets or early detection and monitoring of biomarkers are needed to prevent PVR. Inflammatory factors and retinal imaging could be valuable in monitoring the treatment effect. However, the acquisition of vitreous fluids is an invasive procedure. A circulating biomarker may be more accessible. In this study, we aimed to assess not only the growth factors and cytokine profile in serum but also the cytokine profile in the vitreous to discover possible diagnostic markers for evaluating treatment response in PVR patients. 


\section{METHODS}

\section{Subjects}

This is a prospective consecutive case series. This study conformed to the tenets of the Declaration of Helsinki and was approved by the institutional research board of Zhongshan Ophthalmic Center, Sun Yat-sen University. Blood samples for this study were obtained from patients before surgery, and vitreous samples were collected during pars plana vitrectomy in Zhongshan Ophthalmic Center after obtaining informed consent from each patient. Inclusion criteria included all patients undergoing standard three-port pars plana vitrectomy with a single surgeon (Shaochong Zhang) for indications of primary RRD or primary PVR (no prior surgery for RRD) secondary to RRD and epiretinal membranes (EM) or macular hole $(\mathrm{MH})$. Patients with EM or MH were used as control. Patients with systemic diseases (i.e., diabetes, immunologic diseases, infections), uveitis, glaucoma, or any other conditions that might influence the cytokine levels in serum or vitreous were excluded. The extent of PVR was classified according to the Retina Society Terminology Committee, which grades the appearance of PVR into four groups [11]. Primary RRD was defined as early PVR (stage A and B), which combined mild vitreous opacities, retinal stiffening, and curled hole edges.

\section{Collection of Human Samples}

For serum preparation, $5 \mathrm{ml}$ peripheral blood was collected into EDTA tubes before surgery, kept still for over $30 \mathrm{~min}$, and then centrifuged at $3000 \mathrm{rpm}$ for $10 \mathrm{~min}$ at $4{ }^{\circ} \mathrm{C}$. The supernatant was transferred into a sterile cryogenic vial. All the serum specimens were frozen $\left(-80^{\circ} \mathrm{C}\right)$ until use.

For vitreous preparation, 200-500 $\mu \mathrm{l}$ undiluted vitreous fluid was collected at the time of pars plana vitrectomy, depending on the different intraocular pressure of each patient. The vitreous fluid was collected with a 2-ml syringe before intraocular infusion using a vitreous cutter. All vitreous specimens were placed on ice right away and transferred into sterile tubes. After centrifugation for $10 \mathrm{~min}$ at $4{ }^{\circ} \mathrm{C}$ to avoid red blood cells or other confounding cell material, the vitreous sample was transferred to another sterile tube and kept at $-80^{\circ} \mathrm{C}$ until assay.

\section{Inflammation-Related Factor Analysis}

All the samples were detected using a MILLIPLEX MAP Human Cytokine/Chemokine Magnetic Bead Panel (Millipore-HCYTMAG-60KPX38) on a Luminex platform according to the manufacturer's instructions. Twenty-five microliters of serum or vitreous fluid per well was used for analysis. Thirty-one and ten different analytes were examined in serum and vitreous samples, respectively.

Table 1 Characteristics of the study population

\begin{tabular}{llll}
\hline Serum sample & Control (MH/EM) & RRD & PVR \\
\hline Number of patients & 13 & 11 & 12 \\
Age (mean \pm SD) & $64.38 \pm 6.90$ & $44.64 \pm 14.08$ & $56.00 \pm 11.68$ \\
Gender & & & \\
Female & $9,69 \%$ & $3,27 \%$ & $2,17 \%$ \\
Male & $4,31 \%$ & $8,73 \%$ & $10,83 \%$ \\
Duration of the symptoms (days) mean (range) & $488.46(30-1460)$ & $17.09(7-60)$ & $77.00(10-210)$ \\
PVR grade (range) & - & A-B & C-D \\
\hline
\end{tabular}

$M H$ macular hole, $E M$ epiretinal membrane, $P V R$ proliferative vitreoretinopathy, $R R D$ rhegmatogenous retinal detachment 
Table 2 Overview of 31 inflammatory factors from serum samples

\begin{tabular}{|c|c|c|c|c|c|}
\hline & $\begin{array}{l}\text { Control }(\mathrm{pg} / \mathrm{ml}) \\
\text { mean } \pm \mathrm{SD}\end{array}$ & $\begin{array}{l}\mathrm{RRD}(\mathrm{pg} / \mathrm{ml}) \\
\text { mean } \pm \mathrm{SD}\end{array}$ & $\begin{array}{l}\operatorname{PVR}(\mathrm{pg} / \mathrm{ml}) \\
\text { mean } \pm \mathrm{SD}\end{array}$ & $\boldsymbol{F}$ & $\begin{array}{l}p \text { value } \\
\text { (intergroup) }\end{array}$ \\
\hline EGF & $143.35 \pm 93.59$ & $156.00 \pm 89.80$ & $142.58 \pm 82.16$ & 0.082 & 0.92 \\
\hline FGF-2 & $108.22 \pm 105.77$ & $75.99 \pm 32.59$ & $63.28 \pm 24.91$ & 1.47 & 0.25 \\
\hline $\mathrm{TGF} \alpha$ & $6.24 \pm 4.87$ & $8.94 \pm 5.33$ & $7.04 \pm 4.86$ & 0.90 & 0.42 \\
\hline VEGF & $179.31 \pm 189.48$ & $268.59 \pm 403.13$ & $84.90 \pm 109.75$ & 1.30 & 0.29 \\
\hline $\mathrm{TNF} \alpha$ & $21.60 \pm 9.89$ & $19.28 \pm 9.19$ & $20.08 \pm 6.62$ & 0.22 & 0.80 \\
\hline Eotaxin & $126.09 \pm 53.16$ & $109.18 \pm 67.44$ & $124.08 \pm 60.79$ & 0.27 & 0.77 \\
\hline GM-CSF & $1.65 \pm 0.72$ & $1.35 \pm 0.60$ & $2.37 \pm 4.56$ & 0.45 & 0.64 \\
\hline IFN $\alpha 2$ & $0.87 \pm 1.76$ & $3.46 \pm 4.91$ & $3.35 \pm 6.74$ & 1.13 & 0.33 \\
\hline IFN $\gamma$ & $2.60 \pm 4.22$ & $1.53 \pm 0.79$ & $5.69 \pm 8.15$ & 1.86 & 0.17 \\
\hline GRO & $876.11 \pm 223.72$ & $1094.18 \pm 370.09$ & $813.84 \pm 246.69$ & 3.13 & 0.06 \\
\hline MDC & $985.78 \pm 221.09$ & $1013.55 \pm 343.62$ & $923.59 \pm 465.64$ & 0.18 & 0.83 \\
\hline sCD40L & $6289.78 \pm 2387.03$ & $7584.09 \pm 3110.73$ & $6756.08 \pm 1632.71$ & 0.86 & 0.43 \\
\hline IP-10 & $178.55 \pm 85.40$ & $124.36 \pm 58.95$ & $155.25 \pm 45.22$ & 2.00 & 0.15 \\
\hline MCP-1 & $608.95 \pm 142.99$ & $456.12 \pm 156.01$ & $517.05 \pm 126.18$ & 3.12 & 0.06 \\
\hline MIP-1 $1 \alpha$ & $7.47 \pm 11.66$ & $3.17 \pm 4.19$ & $6.64 \pm 5.81$ & 0.91 & 0.41 \\
\hline MIP-1 $\beta$ & $50.03 \pm 26.58$ & $47.34 \pm 31.35$ & $53.03 \pm 31.22$ & 0.11 & 0.90 \\
\hline IL-1 $1 \alpha$ & $0.20 \pm 0.21$ & $0.13 \pm 0.09$ & $0.18 \pm 0.30$ & 0.26 & 0.77 \\
\hline IL-1 $\beta$ & $1.00 \pm 0.53$ & $0.85 \pm 0.34$ & $1.32 \pm 1.72$ & 0.58 & 0.57 \\
\hline IL-2 & $1.18 \pm 0.22$ & $1.18 \pm 0.22$ & $1.73 \pm 1.83$ & 1.06 & 0.36 \\
\hline IL-3 & $0.45 \pm 0.19$ & $0.43 \pm 0.08$ & $0.38 \pm 0.04$ & 0.94 & 0.40 \\
\hline IL-4 & $2.21 \pm 5.30$ & $3.65 \pm 5.18$ & $1.19 \pm 2.95$ & 0.83 & 0.45 \\
\hline IL-5 & $0.76 \pm 0.21$ & $0.62 \pm 0.17$ & $0.69 \pm 0.32$ & 1.07 & 0.36 \\
\hline IL-6 & $0.57 \pm 0.88$ & $0.27 \pm 0.23$ & $0.88 \pm 2.15$ & 0.60 & 0.56 \\
\hline IL-7 & $2.06 \pm 1.41$ & $2.90 \pm 3.49$ & $1.61 \pm 0.63$ & 1.08 & 0.35 \\
\hline IL-8 & $9.81 \pm 8.38$ & $6.55 \pm 5.97$ & $8.04 \pm 14.47$ & 0.30 & 0.74 \\
\hline IL-9 & $0.46 \pm 0.21$ & $0.45 \pm 0.19$ & $0.48 \pm 0.27$ & 0.06 & 0.95 \\
\hline IL-10 & $2.51 \pm 5.44$ & $1.88 \pm 3.96$ & $0.53 \pm 0.15$ & 1.21 & 0.31 \\
\hline IL-12 p70 & $2.03 \pm 4.33$ & $0.72 \pm 1.34$ & $0.61 \pm 0.43$ & 1.05 & 0.36 \\
\hline IL-13 & $0.22 \pm 0.29$ & $0.18 \pm 0.28$ & $0.10 \pm 0.07$ & 0.79 & 0.46 \\
\hline IL-15 & $1.56 \pm 0.75$ & $1.18 \pm 0.31$ & $1.34 \pm 0.40$ & 1.50 & 0.24 \\
\hline IL-17A & $11.95 \pm 23.61$ & $4.70 \pm 7.66$ & $3.00 \pm 3.41$ & 1.26 & 0.30 \\
\hline
\end{tabular}

$R R D$ rhegmatogenous retinal detachment, $P V R$ proliferative vitreoretinopathy 
Table 3 Characteristics of the study population

\begin{tabular}{lll}
\hline Vitreous sample & Control (MH/EM) & PVR \\
\hline Number of patients & 10 & 24 \\
Age (mean \pm SD) & $63.40 \pm 5.83$ & $49.12 \pm 16.03$ \\
Gender & & \\
Female & $6,50 \%$ & $9,38 \%$ \\
Male & $6,50 \%$ & $15,62 \%$ \\
Duration of the symptoms (days) mean (range) & $376.50(30-1460)$ & $51.38(4-240)$ \\
PVR grade (range) & - & $C-D$ \\
\hline
\end{tabular}

$M H$ macular hole, $E M$ epiretinal membrane, $P V R$ proliferative vitreoretinopathy

\section{Statistical Analysis}

SPSS (version 25.0) was used for all statistical analyses. The box plot was used to describe the dispersion of each group. Statistical significance was determined by independent sample $t$-test between two groups or by one-way analysis of variance (ANOVA) among three groups. To determine whether the data were normally distributed, we used the Shapiro-Wilk test. We used post-hoc Fisher's least significant difference (LSD) for normally distributed data. If the data were not normally distributed, the posthoc Tamhane's T2 was used. $P<0.05$ was considered statistically significant. Extreme outliers ( $>$ upper quartile $+3 \times$ interquartile range) were excluded from statistical analysis.

\section{RESULTS}

\section{Comparisons of Circulating Inflammation-Related Factor Profiles}

In total, 36 serum samples were obtained from patients. Patients with MH or EM were chosen as the control group. Patient characteristics are listed in Table 1 . To analyze the different growth factors and cytokine profiles between RRD without PVR and severe PVR, we divided patients into three groups (Table 1). Four different growth factors, 12 non-interleukin cytokines, and 15 interleukins were analyzed and compared in the serum sample of the RRD, PVR, and control group (MH or EM) patients (Table 2). None of the 31 factors were found to be significantly different, which indicated that RRD and secondary PVR might be a local inflammation.

\section{Comparisons of Intravitreal Inflammation-Related Factor Profiles}

To further study the abnormal intravitreal inflammatory factor profiles in the vitreous of patients with severe PVR, 34 vitreous samples were obtained from patients with $\mathrm{MH}, \mathrm{EM}$, and PVR. Patient characteristics are listed in Table 3.

Five different growth factors and five cytokines were measured in the vitreous samples. These factors were involved in the cell proliferation, EMT process, and inflammation. The concentration levels of PDGF-AA, TGF- $\alpha$, VEGF, IL-6, IL-8, and TNF $\beta$ (Table 4) were significantly increased in the vitreous of PVR patients compared with $\mathrm{MH}$ or EM patients (Figs. 1, 2).

\section{DISCUSSION}

Previous studies have documented circulating monocytes extravasated rapidly from the vasculature and lining the vitreal surface of the retina following induced RD [12, 13]. Monocyte/macrophage infiltration and activity 
Table 4 Overview of ten inflammatory factors from vitreous samples

\begin{tabular}{lccc}
\hline & Control $(\mathbf{p g} / \mathbf{m l})$ mean \pm SD & PVR $(\mathbf{p g} / \mathbf{m l})$ mean \pm SD & $p$ value \\
\hline EGF & $0.78 \pm 0.28$ & $1.01 \pm 0.57$ & 0.23 \\
FGF-2 & $5.91 \pm 0.99$ & $6.27 \pm 1.14$ & 0.39 \\
PDGF-AA & $64.22 \pm 59.82$ & $179.90 \pm 151.92$ & 0.027 \\
TGF $\alpha$ & $0.96 \pm 0.14$ & $3.07 \pm 2.19$ & 0.00 \\
VEGF & $3.22 \pm 0.34$ & $5.54 \pm 4.32$ & 0.015 \\
IL-1 $\beta$ & $0.29 \pm 0.053$ & $0.32 \pm 0.037$ & 0.10 \\
IL-6 & $1.25 \pm 2.50$ & $13.38 \pm 18.19$ & 0.004 \\
IL-8 & $2.81 \pm 3.85$ & $18.75 \pm 17.37$ & 0.00 \\
TNF $\alpha$ & $0.77 \pm 0.061$ & $1.12 \pm 0.81$ & 0.19 \\
TNF $\beta$ & $0.18 \pm 0.013$ & $0.19 \pm 0.015$ & 0.038 \\
\hline
\end{tabular}

PVR proliferative vitreoretinopathy
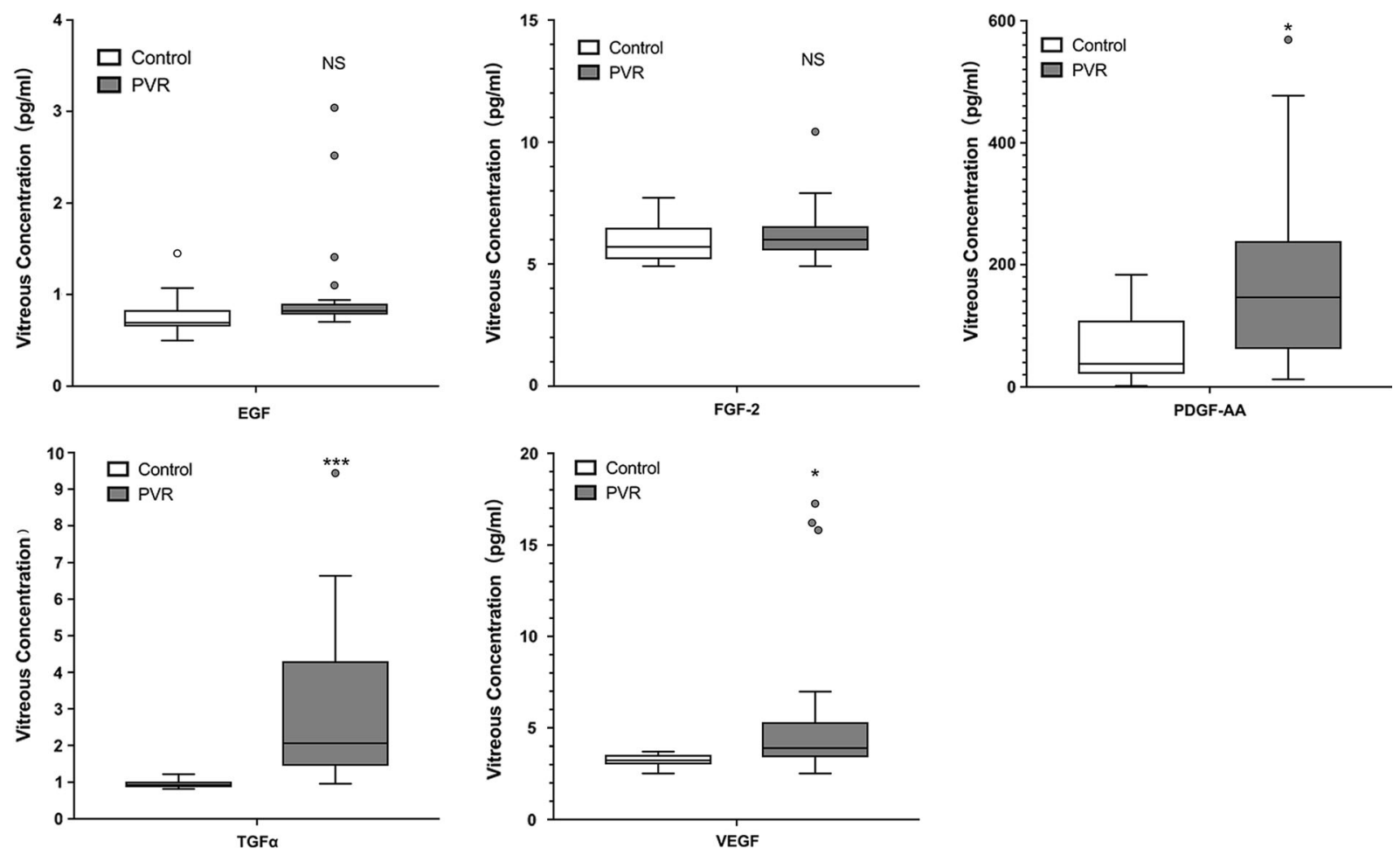

Fig. 1 Five different growth factors were measured in the vitreous fluid samples. PDGF-AA, TGF- $\alpha$, and VEGF were significantly increased in the vitreous fluid of

proliferative vitreoretinopathy (PVR) patients compared with macular hole $(\mathrm{MH})$ or epiretinal membrane (EM) patients. ${ }^{*} p<0.05,{ }^{* * *} p<0.001, N S$ not significant 

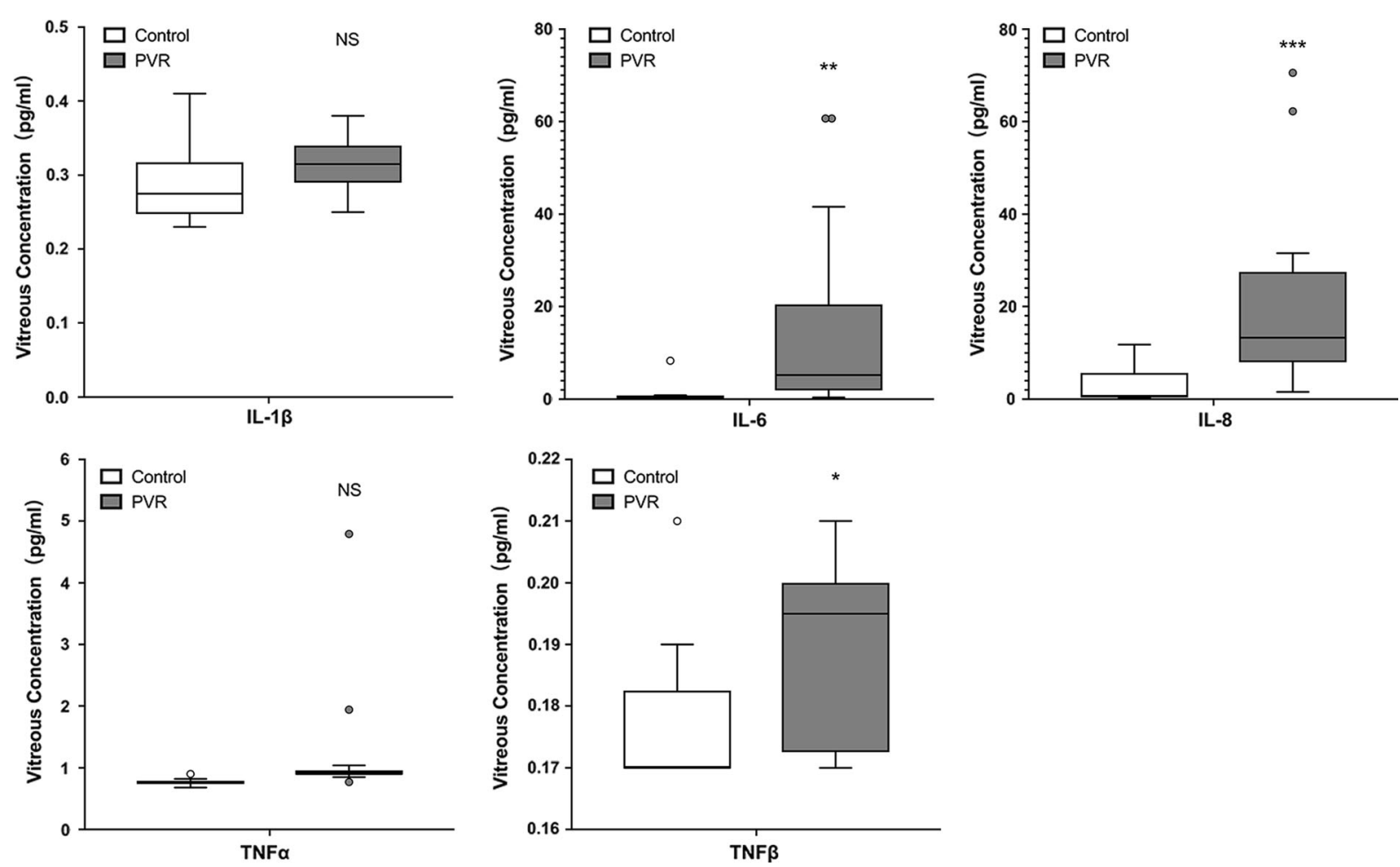

Fig. 2 Five different cytokines were measured in the vitreous fluid samples. IL-6, IL-8, and TNF $\beta$ were significantly increased in the vitreous fluid of proliferative

vitreoretinopathy (PVR) patients compared with macular hole $(\mathrm{MH})$ or epiretinal membrane (EM) patients. ${ }^{*} p<0.05,{ }^{* *} p<0.01,{ }^{* * *} p<0.001, N S$ not significant

contributed to inflammation in the progress of PVR. In our study, 31 inflammation-related factors were all found to be insignificantly different in the blood of RRD and PVR patients compared with controls, indicating that no circulating biomarkers could help predict PVR from an early stage of RRD. RRD and secondary PVR might be a local "inflammatory condition." In our collected vitreous samples, six of the ten measured inflammation-related factors were significantly upregulated in PVR patients. As a supplement to previous research works, the concentrations of TGF- $\alpha$ and TNF $\beta$ were found upregulated in our study. The evolution of RRDinduced PVR involved a tissue trauma repair process, which is triggered by the detachment of neuroretina [2]. The photoreceptors started degeneration as soon as the detachment and continued remodeling or apoptosis as long as the photoreceptor layer was detached [14]. In addition, Müller cells, endothelial cells, microglia, astrocytes, and pericytes start to proliferate after retinal detachment [15]. Previous studies

indicated that RRD could lead to significant upregulation in a number of growth factors and cytokines in the vitreous. These factors mediated the retinal trauma repair process, which results in PVR. Therefore, finding a circulating biomarker can facilitate the monitoring of the therapeutic effect of the surgery and the prevention of PVR.

Retinal diseases, such as diabetic retinopathy (DR), have been proved to have specific important circulating inflammatory factors. Studies have proved that diabetic macular edema (DME) results from both systemic and local inflammation [16]. TNF $\alpha$ was proposed as an important circulating inflammatory factor in serum in DR [17]. The TNF $\alpha$ level was associated with the risk of non-proliferative DR and DME $[18,19]$. IL-6 was also upregulated in both serum and vitreous fluid, which was correlated with DME and progressions of DR [20, 21]. In this study, we aimed to discovered a specific circulating inflammatory factor to predict the severity of the PVR just like the findings in PDR- 
related studies, which would provide new clinical indicators for the evaluation of the therapeutic effect of PVR. However, we found that the RRD or primary PVR did not affect the levels of inflammatory factors in the blood. This is probably because PDR is a systemic chronic disease and PVR is a local acute inflammation.

Levels of PDGF-AA, TGF- $\alpha$, VEGF, IL-6, IL-8, and TNF $\beta$ were significantly increased in the vitreous of PVR patients in our study. While the other four inflammatory factors (including EGF, FGF-2, IL-1 $\beta$, and TNF $\alpha$ ) were not significantly increased, none were decreased. Among them, PDGF, VEGF, IL-6, and IL-8 have been reported to increase in PVR patients. Like in previous studies, TGF- $\alpha$ and TNF $\beta$ were found upregulated in our study. TGF- $\alpha$ is a member of the EGF family, and it binds to EGF receptor (EGFR). TGF- $\alpha$ is reported to be involved in keratinocytes or neural cell proliferation [22, 23], prostate tumorigenesis [24], and angiogenesis [25]. TGF- $\alpha$ enhanced corneal epithelial cell migration by promoting the internalization of EGFR [22]. TGF- $\alpha$ induced EMT in prostate cancer cells [24]. Consistently, this evidence suggests that TGF- $\alpha$ might play a role in PVR formation by promoting both migration and EMT. Secreted by lymphocytes, TNF $\beta$ is a member of the TNF superfamily and homologous with TNF $\alpha$ [26]. TNF $\beta$ is involved in proinflammatory cascade signaling and apoptotic pathways, which play an important role in inflammatory joint diseases $[27,28]$. In our research, TGF- $\alpha$ and TNF $\beta$ were significantly upregulated in PVR patients compared with controls, which indicated that TGF- $\alpha$ and TNF $\beta$ might participate in the PVR process. Further experiments are needed to clarify the role of TGF- $\alpha$ and TNF $\beta$ in PVR.

\section{CONCLUSIONS}

In summary, our findings show that all the inflammation-related factors were not significantly different in serum, indicating that PVR was only a local retinal inflammation. Adding to previous research, the concentrations of PDGF-AA, TGF- $\alpha$, VEGF, IL-6, IL- 8 , and TNF $\beta$ significantly increased in the vitreous of PVR patients.

\section{ACKNOWLEDGEMENTS}

Funding. This study was supported by the Fundamental Research Funds of the State Key Laboratory of Ophthalmology (30306020240020312) and Natural Science Foundation of Guangdong Province of China (2019A1515010189). No funding or sponsorship was received for the rapid service fees of this article. The open access fees were funded by the study sponsor.

Authorship. All named authors meet the International Committee of Medical Journal Editors (ICMJE) criteria for authorship for this article, take responsibility for the integrity of the work as a whole, and have given their approval for this version to be published.

Disclosures. Yao Ni, Yingyan Qin, Zijing Huang, Fangyuan Liu, Shaochong Zhang, and Zhaotian Zhang declare that they have no conflict of interest.

Compliance with Ethics Guidelines. The study was approved by the Institutional Review Board of Zhongshan Ophthalmic Center (ZOC) affiliated to Sun Yat-sen University (Guangzhou, China) and performed in accordance with the 1964 Helsinki Declaration and its later amendments or comparable ethical standards. Informed consent was obtained from all individual participants included in the study.

Data Availability. The datasets generated during and/or analyzed during the current study are available from the corresponding author on reasonable request.

Open Access. This article is licensed under a Creative Commons Attribution-NonCommercial 4.0 International License, which permits any non-commercial use, sharing, adaptation, distribution and reproduction in any medium or format, as long as you give appropriate credit 
to the original author(s) and the source, provide a link to the Creative Commons licence, and indicate if changes were made. The images or other third party material in this article are included in the article's Creative Commons licence, unless indicated otherwise in a credit line to the material. If material is not included in the article's Creative Commons licence and your intended use is not permitted by statutory regulation or exceeds the permitted use, you will need to obtain permission directly from the copyright holder. To view a copy of this licence, visit http://creativecommons.org/licenses/by$\mathrm{nc} / 4.0 /$.

\section{REFERENCES}

1. Kwon OW, Song JH, Roh MI. Retinal detachment and proliferative vitreoretinopathy. Dev Ophthalmol. 2016;55:154-62.

2. Pastor JC, Rojas J, Pastor-Idoate S, Di Lauro S, Gonzalez-Buendia L, Delgado-Tirado S. Proliferative vitreoretinopathy: a new concept of disease pathogenesis and practical consequences. Prog Retin Eye Res. 2016;51:125-55.

3. Takahashi S, Adachi K, Suzuki Y, Maeno A, Nakazawa M. Profiles of inflammatory cytokines in the vitreous fluid from patients with rhegmatogenous retinal detachment and their correlations with clinical features. Biomed Res Int. 2016;2016: 4256183.

4. Hoerster R, Fauser S, Cursiefen C, Kirchhof B, Heindl LM. The influence of systemic renin-angiotensin-inhibition on ocular cytokines related to proliferative vitreoretinopathy. Graefe's Arch Clin Exp Ophthalmol. 2017;255(9):1721-5.

5. Pollreisz A, Sacu S, Eibenberger K, et al. Extent of detached retina and lens status influence intravitreal protein expression in rhegmatogenous retinal detachment. Invest Ophthalmol Vis Sci. 2015;56(9):5493-502.

6. Si Y, Wang J, Guan J, Han Q, Hui Y. Platelet-derived growth factor induced alpha-smooth muscle actin expression by human retinal pigment epithelium cell. J Ocul Pharmacol Ther. 2013;29(3):310-8.

7. Nakazawa T, Matsubara A, Noda K, et al. Characterization of cytokine responses to retinal detachment in rats. Mol Vis. 2006;12:867-78.
8. Nakazawa T, Kayama M, Ryu M, et al. Tumor necrosis factor-alpha mediates photoreceptor death in a rodent model of retinal detachment. Invest Ophthalmol Vis Sci. 2011;52(3):1384-91.

9. Garweg JG, Zandi S, Pfister I, Rieben R, Skowronska $\mathrm{M}$, Tappeiner C. Cytokine profiles of phakic and pseudophakic eyes with primary retinal detachment. Acta Ophthalmol. 2019;97(4):e580-e588588.

10. Pennock S, Haddock LJ, Eliott D, Mukai S, Kazlauskas A. Is neutralizing vitreal growth factors a viable strategy to prevent proliferative vitreoretinopathy? Prog Retin Eye Res. 2014;40:16-34.

11. Hilton G, Machemer R, Michels R, Okun E, Schepens $C$, Schwartz A. The classification of retinal detachment with proliferative vitreoretinopathy. Ophthalmology. 1983;90(2):121-5.

12. Okunuki Y, Mukai R, Pearsall EA, et al. Microglia inhibit photoreceptor cell death and regulate immune cell infiltration in response to retinal detachment. Proc Natl Acad Sci U S A. 2018;115(27):E6264-E62736273.

13. Wang X, Miller EB, Goswami M, et al. Rapid monocyte infiltration following retinal detachment is dependent on non-canonical IL6 signaling through gp130. J Neuroinflammation. 2017;14(1): 121.

14. Fisher SK, Lewis GP. Muller cell and neuronal remodeling in retinal detachment and reattachment and their potential consequences for visual recovery: a review and reconsideration of recent data. Vision Res. 2003;43(8):887-97.

15. Lewis GP, Charteris DG, Sethi CS, Leitner WP, Linberg KA, Fisher SK. The ability of rapid retinal reattachment to stop or reverse the cellular and molecular events initiated by detachment. Invest Ophthalmol Vis Sci. 2002;43(7):2412-20.

16. Figueras-Roca M, Molins B, Sala-Puigdollers A, et al. Peripheral blood metabolic and inflammatory factors as biomarkers to ocular findings in diabetic macular edema. PLoS ONE. 2017;12(3):e0173865.

17. Preciado-Puga MC, Malacara JM, Fajardo-Araujo $\mathrm{ME}$, et al. Markers of the progression of complications in patients with type 2 diabetes: a one-year longitudinal study. Exp Clin Endocrinol Diabetes. 2014;122(8):484-90.

18. Zorena K, Mysliwska J, Mysliwiec M, et al. Serum TNF-alpha level predicts nonproliferative diabetic retinopathy in children. Mediators Inflamm. 2007;2007:92196.

19. Kocabora MS, Telli ME, Fazil K, et al. Serum and aqueous concentrations of inflammatory markers 
in diabetic macular edema. Ocul Immunol Inflamm. 2016;24(5):549-54.

20. Patel JI, Saleh GM, Hykin PG, Gregor ZJ, Cree IA. Concentration of haemodynamic and inflammatory related cytokines in diabetic retinopathy. Eye. $2008 ; 22(2): 223-8$.

21. Shimizu E, Funatsu H, Yamashita H, Yamashita T, Hori S. Plasma level of interleukin-6 is an indicator for predicting diabetic macular edema. Jpn J Ophthalmol. 2002;46(1):78-83.

22. McClintock JL, Ceresa BP. Transforming growth factor-\{alpha\} enhances corneal epithelial cell migration by promoting EGFR recycling. Invest Ophthalmol Vis Sci. 2010;51(7):3455-61.

23. Dai $\mathrm{X}$, Chen J, Xu F, et al. TGFalpha preserves oligodendrocyte lineage cells and improves white matter integrity after cerebral ischemia. J Cereb Blood Flow Metab. 2020;40(3):639-55.

24. Qin W, Pan Y, Zheng X, et al. MicroRNA-124 regulates TGF-alpha-induced epithelial-mesenchymal transition in human prostate cancer cells. Int J Oncol. 2014;45(3):1225-311.

25. Klotzsche-von Ameln A, Prade I, Grosser M, et al. PHD4 stimulates tumor angiogenesis in osteosarcoma cells via TGF-alpha. Mol Cancer Res. 2013;11(11):1337-488.

26. Aggarwal BB, Moffat B, Harkins RN. Human lymphotoxin. Production by a lymphoblastoid cell line, purification, and initial characterization. J Biol Chem. 1984;259(1):686-91.

27. Calmon-Hamaty F, Combe B, Hahne M, Morel J. Lymphotoxin alpha revisited: General features and implications in rheumatoid arthritis. Arthritis Res Ther. 2011;13(4):232.

28. Buhrmann C, Popper B, Aggarwal BB, Shakibaei M. Resveratrol downregulates inflammatory pathway activated by lymphotoxin $\alpha$ (TNF- $\beta$ ) in articular chondrocytes: comparison with TNF- $\alpha$. PLoS ONE. 2017;12(11):e0186993. 